\title{
Dynamic Visual Representation of Clinical Efficacy of Ixekizumab in Psoriasis
}

\author{
Jason E. Hawkes (D) - Kyoungah See - Russel Burge - Stephanie Strakbein • \\ Missy McKean-Matthews · Daniel Saure · Melinda Gooderham • \\ Craig Leonardi
}

Received: February 12, 2021 / Published online: May 29, 2021

(c) The Author(s) 2021

\begin{abstract}
Introduction: Ixekizumab, a high-affinity monoclonal antibody that selectively targets interleukin-17A, is an approved treatment for plaque psoriasis. This study aimed to use animated visualizations as a tool to simplify complex data from ixekizumab clinical trials.

Methods: Animated visualizations were developed to show outcomes from ixekizumab clinical trials and a Bayesian network meta-analysis of 11 approved biologics. The visualizations simultaneously highlighted both aggregate
\end{abstract}

Supplementary Information The online version contains supplementary material available at https:// doi.org/10.1007/s13555-021-00548-2.

J. E. Hawkes $(\varangle)$

Department of Dermatology, University of

California-Davis, Sacramento, CA, USA

e-mail: jehawkes@ucdavis.edu

K. See · R. Burge $\cdot$ S. Strakbein · D. Saure

Eli Lilly and Company, Indianapolis, IN, USA

R. Burge

University of Cincinnati, Cincinnati, OH, USA

M. McKean-Matthews

Syneos Health, Raleigh, NC, USA

M. Gooderham

SKiN Centre for Dermatology, Peterborough, ON, Canada

C. Leonardi

Central Dermatology, St. Louis, MO, USA scores and the individual progression of patients over the course of treatment.

Results: The animations provided key messages and information from the complex data in efficient and scientific ways that were also visually pleasing and simple to understand. The animations highlighted (1) rapid reduction in disease severity from baseline; (2) sustained efficacy of ixekizumab in the treatment of skin and nail psoriasis; (3) side-by-side comparisons of treatment efficacy and clinical improvement across trials; (4) simultaneous visual presentation of individual results with summary response over time; and (5) indirect comparison of relative treatment effects with other biologics based on Bayesian network meta-analysis.

Conclusion: The rapid and sustained efficacy of ixekizumab in the treatment of psoriasis was demonstrated using multiple dynamic visualizations with different clinical endpoints. Animated visualizations provided a simpler and more comprehensive understanding of complex data than conventional static figures.

Keywords: Animated visualization; Biologics; Ixekizumab; Meta-analysis 


\section{Key Summary Points}

Why carry out this study?

Ixekizumab, a high-affinity monoclonal antibody that selectively targets interleukin-17A, is an approved treatment for plaque psoriasis.

This study aimed to use animated visualizations as a tool to simplify complex data from ixekizumab clinical trials.

\section{What was learned from this study?}

Dynamic animated visualizations were developed by applying a combination of statistical methods, design principles, and data science.

The animations highlighted both the aggregate and individual response rates from treatment with ixekizumab and other biologics and showed the indirect comparison of relative treatment effects based on Bayesian network meta-analysis.

Animated visualizations can be used to identify meaningful aspects of clinical trial data, such as individual patient variability and trends during clinical trial testing.

\section{DIGITAL FEATURES}

This article is published with digital features, including a summary slide and video animations to facilitate understanding of the article. To view digital features for this article go to https://doi.org/10.6084/m9.figshare.14518881.

\section{INTRODUCTION}

Plaque psoriasis is a chronic inflammatory disease that causes characteristic scaly plaques on the scalp, buttocks, trunk, and extremities [1] and may increase the risk of other psoriasis-associated conditions such as cardiovascular disease, obesity, diabetes, psoriatic arthritis, and psychological comorbidities [2,3]. Patients with plaque psoriasis may face significant quality-oflife challenges [4], highlighting the need for highly effective long-term treatments [1].

Biologics, such as antibodies that inhibit tumor necrosis factor alpha (TNF- $\alpha$ ) and interleukins (IL)-17 and IL-23, have transformed the treatment landscape of psoriasis compared to traditional topical therapies or phototherapy, which may not adequately control moderate-tosevere disease $[5,6]$. The comparative effectiveness of biologics used to treat psoriasis has been evaluated in head-to-head studies as well as indirect comparisons using network meta-analysis (NMA) [7-11]. Ixekizumab, a high-affinity monoclonal antibody that selectively targets IL$17 \mathrm{~A}$, is an example of one such biologic; it has been studied in head-to-head trials versus etanercept, ustekinumab, adalimumab, and guselkumab [10, 12-14] and demonstrated rapid efficacy, followed by long-term durability of treatment response [7-11]. The efficacy of ixekizumab and other phase III-approved biologics was highlighted in a Bayesian NMA, showing the competitive landscape of psoriasis treatments.

Comparative studies and NMAs generate large amounts of complex data, and static graphs and figures are limited in how they depict changes in data over time, especially as they relate to individual variability. Animated visualizations can be used to present key messages and information from complex data in an efficient and scientific manner that is also visually pleasing and simple to understand. These dynamic animations are more engaging and may appeal to a broader audience with heterogeneous learning styles. Here we present several types of animated visualizations that highlight data on the efficacy of ixekizumab in the treatment of moderate-to-severe psoriasis. 


\section{METHODS}

\section{Studies on Ixekizumab Efficacy}

Clinical efficacy data on ixekizumab and comparator drugs were taken from published studies, including the UNCOVER-2 (NCT01597245), UNCOVER-3 (NCT01646177), IXORA-R (NCT03573323), IXORA-S (NCT02561806), and SPIRIT-H2H (NCT03151551) trials. Details regarding the study design, patient selection, treatment arms, and outcome measures were previously described for all studies [10, 12-14] and are outlined in Electronic Supplementary Material (ESM) Table 1.

\section{Assessments}

The following parameters were evaluated to compare onset, durability of response, and rapidity of skin clearance with biologics.

Mean percentage improvement in Psoriasis Area and Severity Index (PASI) score was assessed for:

- Ixekizumab versus etanercept (integrated UNCOVER-2 and UNCOVER-3) from week 0 to week 12.

- Ixekizumab versus adalimumab from week 0 to week 12 (SPIRIT-H2H is a psoriatic arthritis study with the primary endpoint at week 24; however, only data up to week 12 is presented in this paper for consistency with the other studies).

- Ixekizumab versus guselkumab from week 0 to week 12 (IXORA-R).

- Ixekizumab versus ustekinumab from week 0 to week 12 (IXORA-S).

Static Physician's Global Assessment (sPGA) 0 and sPGA $(0,1)$ response rates were compared between ixekizumab and ustekinumab from week 0 to week 52 (IXORA-S). In UNCOVER-3, percentage improvement in Nail Psoriasis Severity Index (NAPSI) scores from baseline to week 264 was evaluated. After 12 weeks of treatment, patients who received ixekizumab once every 2 weeks entered the long-term extension period; ixekizumab was then administered as open-label treatment once every 4 weeks.

\section{Studies and Procedure for NMA}

Bayesian and frequentist NMAs were conducted to compare the rapidity of response of 11 biologics in the treatment of moderate-to-severe psoriasis in adults with IL-17, IL-23p19, IL-12/ $23 p 40$, and TNF- $\alpha$ (all inhibitors are approved by the U.S. Food and Drug Administration) [8]. A systematic literature review was conducted to identify phase 3 randomized studies of biologics with PASI response rates at weeks 2, 4, 8, and 12 and Dermatology Life Quality Index (DLQI; 0, 1) response rates at week 12 . Relative treatment effects with the biologics studied compared to placebo were evaluated. Details of the 33 studies included for this analysis have been previously described [8]. Data related to DLQI were not included in this paper.

Data were utilized from an NMA on the average treatment response with biologics, based on posterior distribution of the relative treatment effects compared to placebo over 12 weeks [8].

\section{Statistical Methods and Analysis}

The PASI total score and mean percentage improvement were provided by least square means from mixed models using repeated measures (MMRM) for ixekizumab and placebo in the induction period of UNCOVER-3 (Fig. 1). For categorical PASI response rates, the number of patients and percentages were summarized with non-responder imputation to account for missing data. The model included the fixed effects of treatment, study, visit, and the treatment-by-visit interaction, and the fixed covariate of the baseline value. The individual patient records of percentage improvement in PASI score from baseline through week 12 by baseline PASI score are displayed in Video 1 (online version only). PASI baseline score $>40$ is presented in a gray area to capture outliers.

Mean percentage improvements in NAPSI score was shown from baseline to 5 years in the UNCOVER-3 study (induction and long-term extension) using a modified baseline observation carried forward imputation (Fig. 2). Mean NAPSI percentage improvement (see Video 2 
[online version only]) at each planned visit was calculated using MMRM following the models for each study and then plotted as a smoothed line. The smoothed line was generated using Bézier spline to determine mean percentage improvement in PASI/NAPSI score between visits to create the effect of line-filling over time.

For the UNCOVER-2 and UNCOVER-3 trials, the model included factors related to individual studies, such as the fixed covariates of baseline value, treatment, study, visit, and the treatment-by-visit interaction. In the IXORA-R analysis, the model included treatment, pooled center, baseline value, visit, treatment-by-visit interaction, and the baseline value by visit. For the IXORA-S analysis, the model included baseline value, treatment, visit, weight group $(\leq 100 \mathrm{~kg},>100 \mathrm{~kg})$, region group (Western European Union [EU], Eastern EU, and North America), and the treatment-by-weight and treatment-by-region interaction terms. The model for the SPIRIT-H2H study included treatment group, concomitant conventional synthetic disease-modifying anti-rheumatic drug use at baseline, moderate-to-severe plaque psoriasis involvement, visit, baseline value, and baseline-by-visit and treatment-by-visit interactions terms.

For the head-to-head comparison animation (see Video 3 [online version only]), mean percentage improvement in PASI score at each planned visit was calculated using MMRM following the models for each study and then plotted as a smoothed line. Similar to Fig. 2, the smoothed line for Fig. 3 was also generated using Bézier spline.

An animation with two separate components-patient-level data at each planned visit on the left and summary sPGA (0) and sPGA (0, 1) response rates for observed records on the right-is shown in Video 4 (online version only). The lines connecting response rates were smoothed using Bézier curves. Solid lines connected sPGA $(0,1)$ response rates and dashed lines connected sPGA (0) response rates. The left side of the figure shows individual patient records of sPGA at each pre-planned study visit in the 52-week treatment period. Patient records were arranged into columns by sPGA value (0-5) and by treatment arm, which was also designated by color. Observed records were denoted by a solid circle in the color of the respective treatment arm. Patients who discontinued treatment were denoted by a solid black circle in the column of their respective prior treatment and their SPGA position was determined using mBOCF methodology. Patients who missed a planned visit, but did not discontinue from the study, appeared as a hollow black circle at the missing visit and were positioned according to their last observed sPGA value until the next observed visit occurred. The radius of the patients' circles were scaled proportionately to the inverse of the sample size by treatment arm to make the relative heights of the dot-columns representative of the proportion of patients in the treatment arm achieving the respective endpoint. Without adjustment, unequal sample sizes in treatment arms bias the impact of the stacked circles when presented side by side. Movement of the patients' dots between sPGA columns was controlled by a Bézier curve drawn between the coordinates of the proceeding and subsequent positions of the dots at planned visits.

A representative sample of posterior treatment effects was generated at each time point and treatment effects from this sample were interpolated using a Bézier spline function to create smooth transitions between posterior distribution curves at each time point (see Video 5 [online version only]; ESM Fig. 2). Himalayan and ridgeline curves that were farther along on the $x$-axis (right-hand side) represented a greater estimated treatment effect. Taller and narrow curves represented more reliable estimates of response rates compared to shorter and wider curves.

Figures 1, 2, 3, 5 and ESM Fig. 2 were created using R versions 3.6.0, grid 3.6.0 (R Development Core Team 98-2013; R Foundation for Statistical Computing, Vienna, Austria ), gridExtra 2.3 [15], plyr 1.8.4 [16]. Figure 4 was created using D3.js (Bostock 2019).

The Bayesian NMA results were obtained based on Bayesian Tool for Meta-Analysis of Networks (rjags 4-9 via R) with fixed-treatment effect and random-baseline effect (ESM Fig. 1). Deviance is a goodness-of-fit statistic and was 
used as a model for hypothesis testing. Normal independent models and model fit were assessed using the deviance information criterion and residual deviance.

This article is based on previously conducted studies and does not contain any new studies with human participants or animals performed by any of the authors.

\section{RESULTS}

\section{Rapid Onset of Response Compared to Placebo (UNCOVER-3 Trial)}

An animation of patients who received either placebo or ixekizumab was presented in two panels, where each dot is an individual patient (Fig. 1); it showed how PASI response changes from baseline PASI to week 12 PASI based on the patients' baseline scores. At week 0 , the dots reflected the baseline PASI distribution on the horizontal axis. When running the animation, the dots moved to PASI improvement levels (vertical axis) at each time point. PASI improvement endpoints, including PASI 50, 75, 90, and 100 (improvement of 50, 75, 90, and $100 \%$ [complete resolution of disease]) were depicted in a gradation of green with the darkest being $100 \%$. When the animation was paused at week 1 , the mean percentage PASI improvements and mean total score PASI were seen at the top; patients who received ixekizumab had a rapid improvement in PASI scores versus those on placebo, including those who were at the lower end of disease severity in these trials (7.3 and $19.6 \%$ for placebo; 35.1 and $13.6 \%$ for ixekizumab). As the animation played out to week 12, the distribution of dots (patients) reflected the differences in efficacy observed in the underlying clinical trial.

PASI Response at Week 12 (UNCOVER-3)

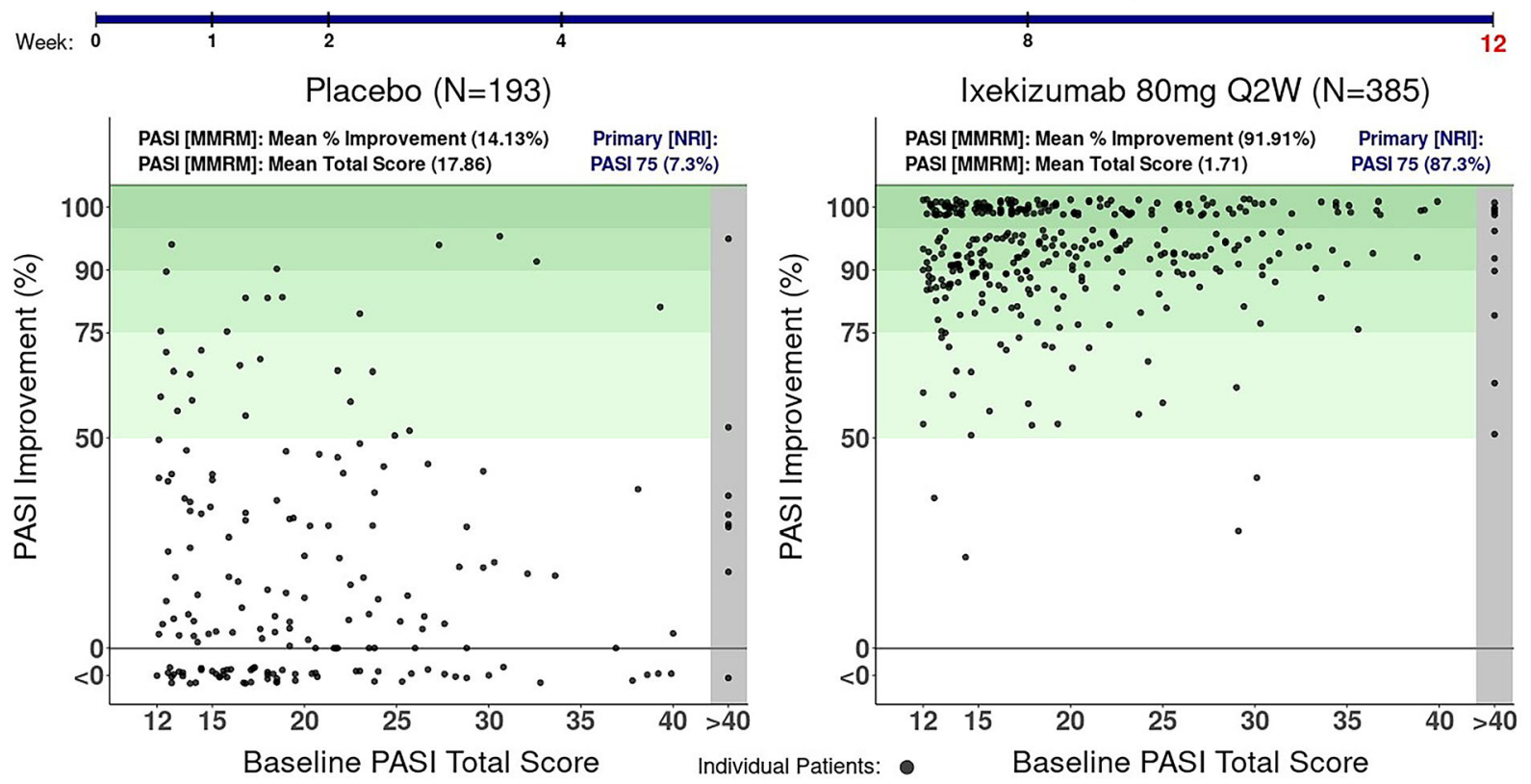

Fig. 1 Skyward plot at week 12. PASI response rates among ixekizumab-treated and placebo-treated patients in the UNCOVER-3 trial. In the skyward scatter plot animation, percentage improvement in PASI scores from baseline through week 12 is highlighted for patients who received either placebo or ixekizumab once every 2 weeks in the UNCOVER-3 trial. MMRM Mixed models using repeated measures, NRI non-responder imputation, $P A S I$ Psoriasis Area and Severity Index, PASI $75 \geq 75 \%$ reduction in PASI scores from baseline 


\section{Improvement in Nail Psoriasis Compared to Placebo (UNCOVER-3 Trial)}

Improvement in nail psoriasis was highlighted in the time-course tornado animation, which included the percentage improvement in NAPSI score from baseline to 5 years as the outcome measure (Fig. 2; also see Video 2 [online version only]). The mean percentage improvements in NAPSI values were presented next to the line for the means and $95 \%$ confidence interval, while the individual patient values were simultaneously displayed by the moving blue dots up to week 264. Based on the animated visualization, patients who received ixekizumab generally maintained high response rates, although they fluctuated briefly for some patients. Most patients consistently achieved complete clearance in the nails, as noted by the cluster of patients in the NAPSI 100 band (darkest green region).

\section{Onset of Action Compared to Other Biologics (Head-to-Head Studies)}

Side-by-side time-course tornado animations (Fig. 3) were used to present data side by side comparing percentage improvement in PASI score from baseline to week 12 between ixekizumab and etanercept (UNCOVER-2 and UNCOVER-3 trials), ixekizumab and ustekinumab (IXORA-S trial), ixekizumab and adalimumab (SPIRIT-H2H trial), and ixekizumab and guselkumab (IXORA-R trial) (see Video 3 [online version only]). Patients who received ixekizumab had higher average PASI percentage improvement than patients who received comparator drugs at all time points within 12 weeks in head-to-head studies. The PASI percentage improvement for individual patients was represented by the blue and orange dots that move up or down as they progress from left to right over time across each figure panel. The

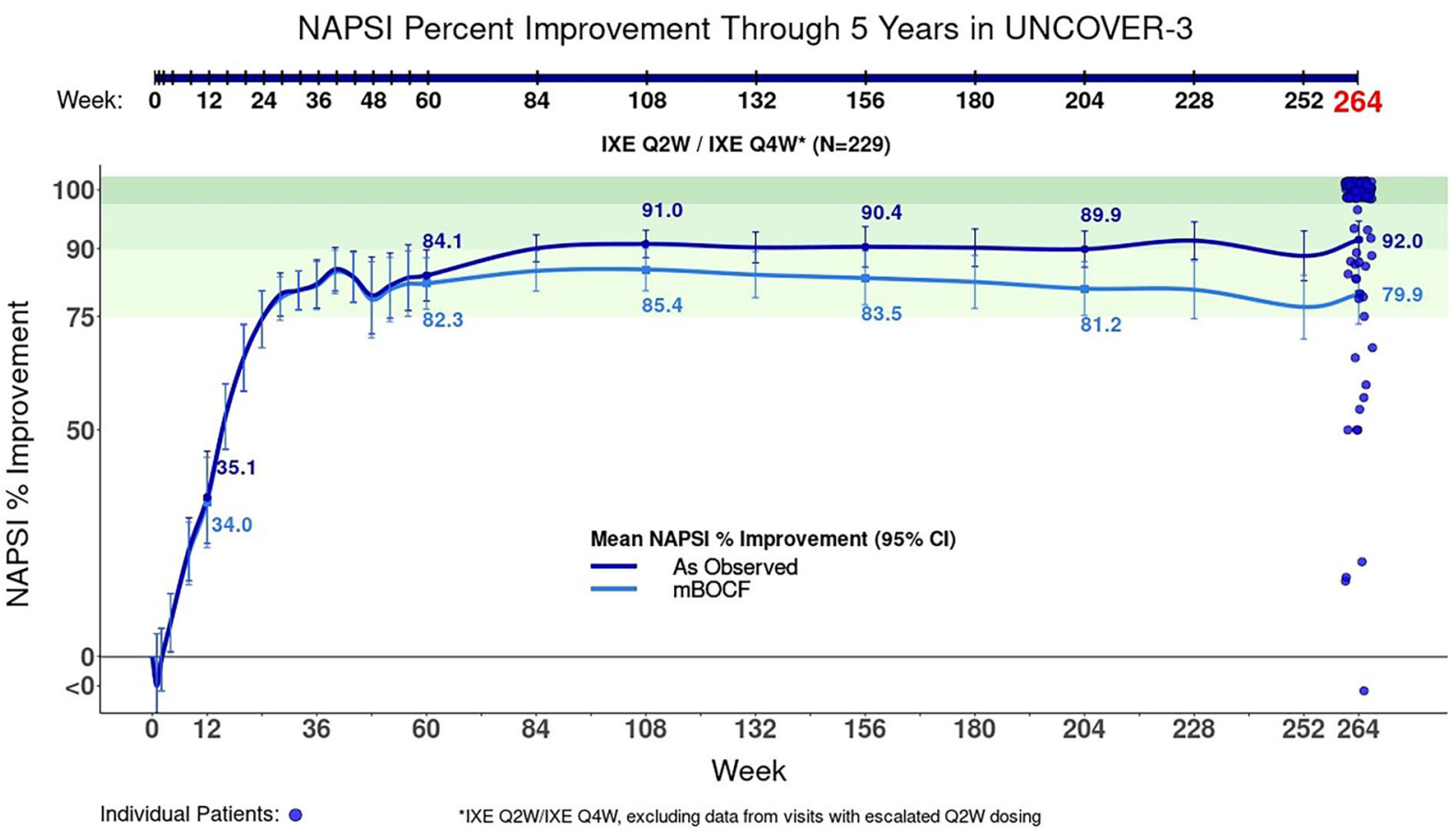

Fig. 2 Time-course tornado plot at week 264. Percentage improvement in NAPSI score in patients treated with IXE in the UNCOVER-3 trial. Percentage improvement in NAPSI score is highlighted in this time-course tornado plot visual animation from the UNCOVER-3 trial.
Patients received IXE once every 2 weeks for 12 weeks $(Q 2 W)$ then IXE once every 4 weeks $(Q 4 W)$ from week 16 to week 264 (excluding titrated visits). IXE Ixekizumab, mBOCF modified baseline observation carried forward, NAPSI Nail Psoriasis Severity Index 

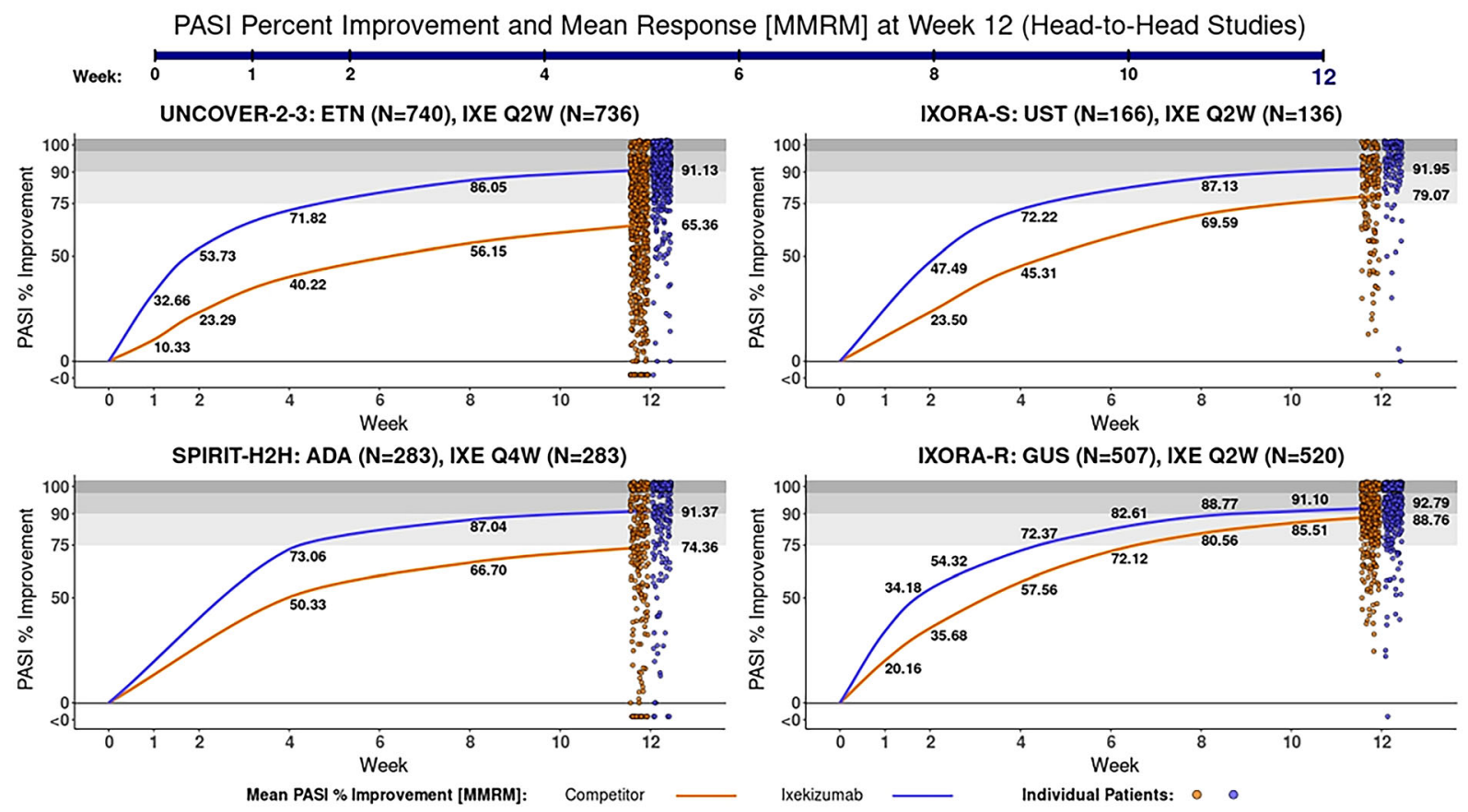

Fig. 3 Side-by-side time-course tornado plot at week 52. Percentage improvement in PASI score in patients treated with IXE and other biologics. In the side-by-side time-course animation, percentage improvement in PASI score is

ixekizumab patients (blue dots) were clustered at generally higher PASI percentage improvement values, which were represented by the gray gradation bands versus the competitor patients (orange dots) who showed more variability in response.

\section{sPGA Response Rates $(0,0 / 1)$ for Ixek- izumab and Ustekinumab (IXORA-S trial)}

Stacked circle animations were used to represent individual sPGA results, alongside the timecourse animation with overall sPGA (0) and sPGA $(0,1)$ response rates for ixekizumab and ustekinumab over 52 weeks (Fig. 4; also see Video 4 [online version only]). On the left side, this animation showed individual patient's sPGA results across time for each time point, with the overall response rate for each sPGA value displayed directly under the stacked circles; the dots moved between the six bars based on their sPGA scores (0-5). The time course on

highlighted in this comparison of treatment response in four psoriasis trials with IXE vs. comparator drugs from baseline through week 52. ADA Adalimumab, ETN etanercept, GUS guselkumab, IXE ixekizumab, UST ustekinumab

the right side showed the summary response rates for two key endpoints: those achieving sPGA (0) and those achieving sPGA $(0,1)$. This type of animation allowed individual and summary data to be displayed simultaneously. For instance, the figure showed that at week 24, $56.6 \%$ of ixekizumab-treated patients achieved sPGA 0 versus $25.0 \%$ of ustekinumab-treated patients. The response for individual patients can be viewed simultaneously with the overall response rate for each treatment group. Scaling the circles to adjust for unequal sample sizes allowed for the stacked circles to be interpreted like a conventional bar chart while still showing how individual patients move to different sPGA classifications over time.

\section{Treatment Effects Compared to Other Biologics (Multiple Studies from the NMA)}

Ridgeline plot animations presented relative treatment effects with biologics compared to 
sPGA Response at Week: 52 (IXORA-S)

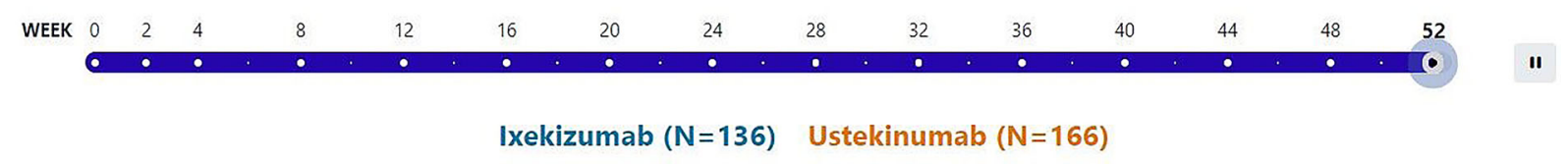

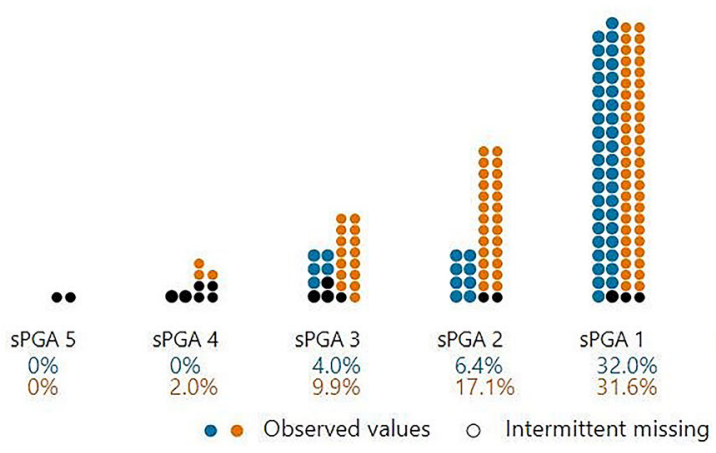

Fig. 4 Simultaneous stacked circle with time-course plot at week 52. sPGA response rates in patients treated with either ixekizumab or ustekinumab. In the stacked circle with time-course plot animation, response rates per the

placebo over time (see Video 5 [online version only]). At week 12, ixekizumab, brodalumab, guselkumab, and risankizumab demonstrated the highest treatment effects for PASI 90 with some overlap as displayed in the animation (Fig. 5). The Himalayan animation simultaneously showed how quickly on average changes occur for treatments in relation to one another, as well as the treatment effect on response rates. This type of animation was useful for making direct and indirect comparisons across multiple compounds and studies, which allowed for comparison without head-to-head data. Ixekizumab and brodalumab treatment groups showed more rapid response rates on PASI 75, and ixekizumab overlapped with risankizumab at week 12 (ESM Fig. 2).

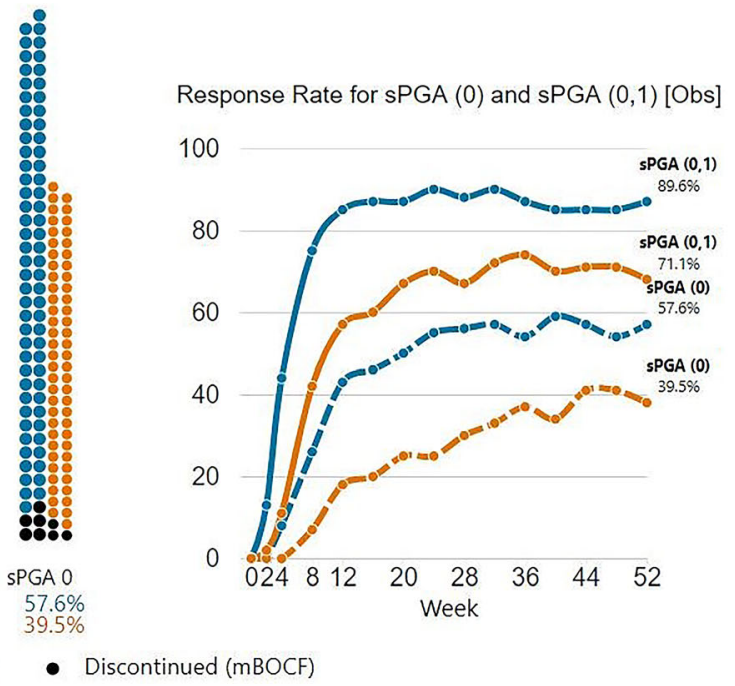

sPGA are shown for patients treated with either ixekizumab or ustekinumab from baseline through week 52 . sPGA Static Physician's Global Assessment, Obs Observed and $m B O C F$ Modified Baseline Carried Forward

\section{DISCUSSION}

Static graphs and figures are limited in depicting how changes in data occur over time. Animated visualization of complex data from head-tohead and NMA psoriasis studies can present more detailed results and may be more engaging for end-users [17]; it also provides an avenue for healthcare providers to explore data trends and to gain clinical insights with increased efficiency.

In our study, we developed dynamic animated visualizations by applying a combination of statistical methods, design principles, and data science. In these novel animations, clinical improvement was observed from week to week for each treatment group-the rate of response was illustrated by the movement of dots 


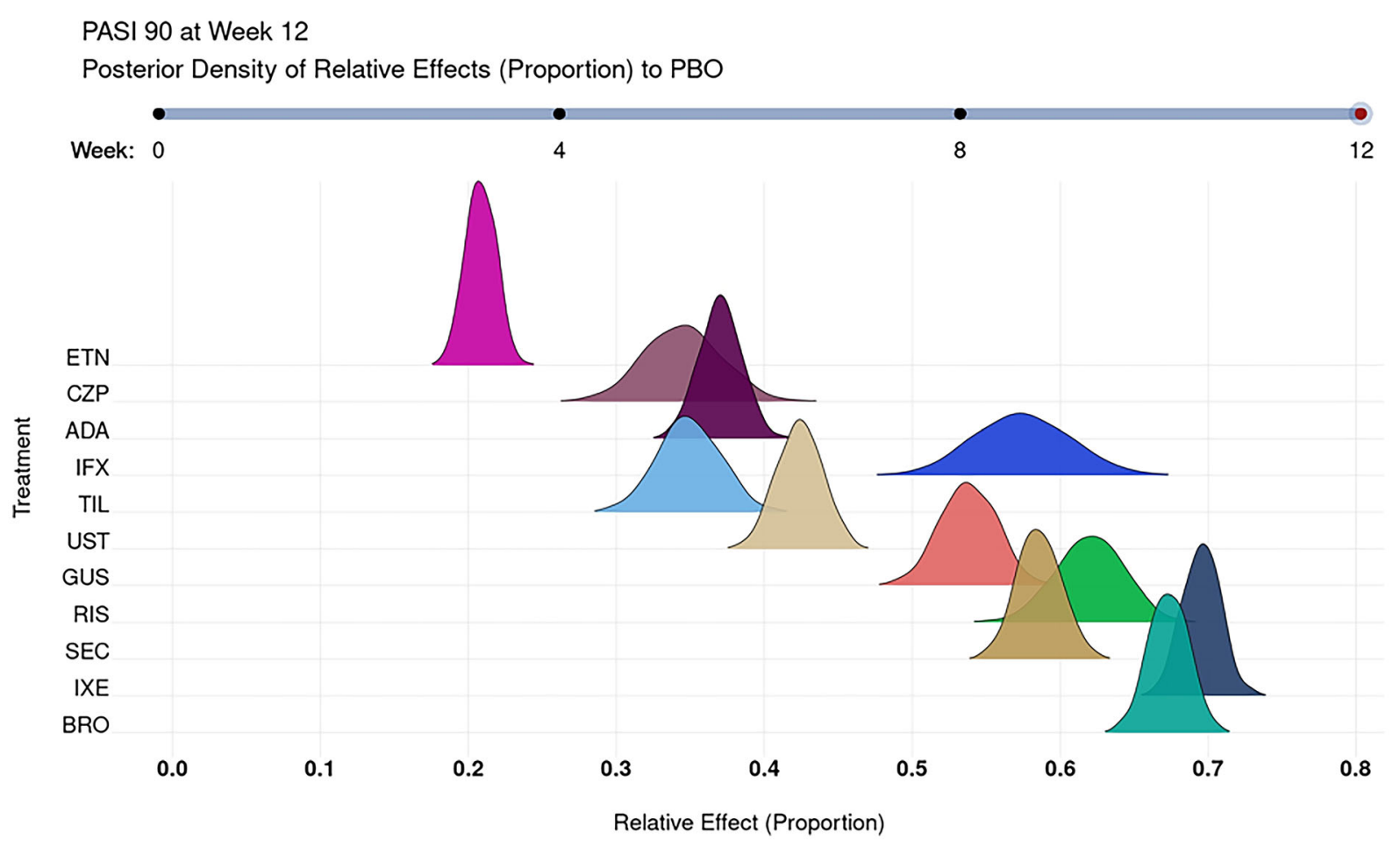

Fig. 5 Ridgeline "Racetrack" plot at week 12. In the ridgeline "racetrack" animation, the competitive landscape of phase 3-approved biologics is shown based on a Bayesian network meta-analysis, which depicts a ridgeline plot with PASI 90 scores through weeks 48-52. BRO Brodalumab, $C Z P$ certolizumab pegol GUS guselkumab, IFX infliximab,

representing patients. The scatterplot animation showed the progress of individual patients, including gains and losses in improvement that occurred from 1 week to the next, providing a more real-world representation of what individuals experience during their psoriasis treatment course. The dots represented percentage improvement in PASI scores among individuals, and the thick clustering of dots indicated that most patients who received ixekizumab had complete clearance. However, in the case of long-term trend analysis, tornado plot animations would be preferable to using a scatterplot.

A tornado time-course plot was used to show sustained efficacy and highlight percentage improvement in NAPSI scores from baseline in patients treated with ixekizumab. After 264 weeks of treatment, both the aggregate and individual response rates were evident. The
$I X E$ ixekizumab, $P B O$ placebo, PASI $90 \geq 90 \%$ improvement in PASI from baseline, RIS risankizumab, SEC secukinumab, TIL tildrakizumab, UST ustekinumab, $E T N$ etanercept, $A D A$ adalimumab

cluster of patients in the dark-green band along the top of the animation showed the proportion of patients who achieved NAPSI 100, which visually highlighted treatment effectiveness week by week and over time.

The psoriasis treatment pipeline is robust, and animations that compare head-to-head studies are useful to demonstrate the mean response to treatment and the overall trends associated with each comparator. In side-byside time-course animations, treatment efficacy at multiple time points can be visually compared across multiple studies. Individual patient data are synced by study time point so that variability trends can be compared both within a study and across studies. The patterns of variability within ixekizumab treatment was consistent in all four studies, while competitors in the same studies had more variable treatment 
responses. The relative efficacy of ixekizumab versus each competitor differed across studies but was shown to be superior in all, as shown by the mean lines.

When ordinal data are available, stacked circle animations can be used to depict overall data trends and individual progress at each time point. However, tracking individual patients' trajectories may be difficult. Therefore, this animation is best used to highlight gross impressions of data variability. Himalayan and ridgeline plots can be used to show the results of Bayesian meta-analysis. The average response to each treatment was calculated relative to placebo and represented as a Himalayan plot or Ridgeline plot. Ridgeline plots are also useful when several treatments are compared, and substantial overlap between treatments is easily visualized.

The natural disease course in psoriasis is more clearly represented using animations than static figures given that dynamic animations highlight disease variations and treatment responses over time. This can be illustrated for specific body sites, such intertriginous skin, scalp, or nails, to provide important clinical insights into differential treatment responses to targeted psoriatic therapies. Many clinical trials and real-world studies have previously shown that special sites of skin (e.g., scalp, nails, joints, palms/soles) do not always respond in line with the observed skin response [18, 19]. Varied individual response could be related to treatment compliance, natural fluctuations in a patient's immune response, and/or external triggers of disease, such as trauma, infections, scratching and stress. Animations can be used as visual aids to help investigators identify interesting clinical observations and formulate hypotheses for further investigation.

The participation of healthcare providers in end-user testing of animated visualizations can confirm their usability and provide information on whether healthcare providers benefit from detailed data displays compared to conventional static presentations [20]. Carefully designed studies or surveys evaluating the users' preferences for these types of novel animations are needed and could directly impact the uptake of clinical information by healthcare providers.
The comparison of clinical trial data should be done carefully due to differences in study design, length of treatment, severity of disease, and patient characteristics. Since IL-17 inhibitors have a faster onset than IL-23 or TNF blockade, the rate of treatment response may appear to be more advantageous at week 12 as treatment difference may not yet be apparent; looking at multiple time points would provide a more comprehensive view of the data. Additionally, while animated visualizations allow for improved understanding of complex data, they are primarily a descriptive instrument of data and, therefore, provide only nominal insights into existing information. Animated visualizations should be used in combination with results that are provided in tables with respective p-values to directly compare psoriasis treatments.

Ixekizumab has demonstrated high efficacy in the treatment of plaque psoriasis based on head-to-head clinical trials with comparator treatments. Data visualization via novel animations is an engaging way to represent findings from clinical trials; this approach may increase efficiency and reduce time spent on data mining. Animated visualizations can be used to identify meaningful aspects of clinical trial data, such as individual patient variability and trends during clinical trial testing. Further investigation of methods to represent clinical trial data is an important endeavor to improve the dissemination and understanding of complex clinical information by healthcare providers.

\section{ACKNOWLEDGEMENTS}

\section{Medical Writing and Editorial Assis-} tance. Writing assistance was provided by Katie Crosslin, PhD, of Evidera/PPD, and Manju Janardhanan, MD, full-time employee of Eli Lilly and Company and was funded by Eli Lilly and Company. Animations support was provided by Nikhil Das Nomula (Fig. 5), a former contracted employee of Eli Lilly and Company. 
Funding. Sponsorship for this study and Rapid Service Fee were funded by Eli Lilly and Company (Indianapolis, IN, USA).

Authorship. All named authors meet the International Committee of Medical Journal Editors criteria for authorship for this article, take responsibility for the integrity of the work as a whole, and have given their approval for this version to be published.

Disclosures. Craig Leonardi has been on an advisory board for, and/or been a consultant for, and/or received speaker's bureau from, and/ or is/was an investigator at the following companies: AbbVie, Actavis, Allergan, Amgen, Boehringer Ingelheim, Celgene, Coherus, Cellceutix, Corrona, Dermira, Eli Lilly and Company, Galderma, Glenmark, Janssen, Leo Pharma, Merck, Novartis, Novella, Ortho Dermatologics, Pfizer, Sandoz, Sienna, Stiefel, Sun Pharmaceuticals, UCB, Vitae, and Wyeth. Melinda Gooderham has been an investigator, speaker and/or advisor for: AbbVie, Amgen, Akros, Arcutis, Bausch Health, Bausch, BMS, Boehringer Ingelheim, Celgene, Dermira, Dermavant, Eli Lilly and Company, Galderma, GSK, Incyte, Janssen, Kyowa Kirin, LEO Pharma, MedImmune, Merck, Novartis, Pfizer, Regeneron, Roche, Sanofi Genzyme, Sun Pharma, and UCB. Jason E. Hawkes currently serves on the medical board of the National Psoriasis Foundation and has been a consultant for AbbVie, Eli Lilly and Company, Janssen, LearnSkin, LEO, Novartis, Pfizer, Regeneron-Sanofi, UCB, VisualDx, and UpToDate. Kyoungah See, Russel Burge, Stephanie Strakbein, Missy McKeanMatthews, and Daniel Saure are employees of Eli Lilly and Company and may be stockholders.

Compliance with Ethics Guidelines. This article is based on previously conducted studies and does not contain any new studies with human participants or animals performed by any of the authors.

Data Availability. All data generated or analyzed during this study are included in this published article/as supplementary information files.

Open Access. This article is licensed under a Creative Commons Attribution-NonCommercial 4.0 International License, which permits any non-commercial use, sharing, adaptation, distribution and reproduction in any medium or format, as long as you give appropriate credit to the original author(s) and the source, provide a link to the Creative Commons licence, and indicate if changes were made. The images or other third party material in this article are included in the article's Creative Commons licence, unless indicated otherwise in a credit line to the material. If material is not included in the article's Creative Commons licence and your intended use is not permitted by statutory regulation or exceeds the permitted use, you will need to obtain permission directly from the copyright holder. To view a copy of this licence, visit http://creativecommons.org/licenses/by$\mathrm{nc} / 4.0 /$.

\section{REFERENCES}

1. Kim WB, Jerome D, Yeung J. Diagnosis and management of psoriasis. Can Fam Phys. 2017;63(4): 278-85.

2. Pariser D, Schenkel B, Carter C, et al. A multicenter, non-interventional study to evaluate patient-reported experiences of living with psoriasis. J Dermatol Treat. 2016;27(1):19-26.

3. Strober B, Karki C, Mason M, et al. Characterization of disease burden, comorbidities, and treatment use in a large, US-based cohort: results from the Corrona Psoriasis Registry. J Am Acad Dermatol. 2018;78(2):323-32.

4. Kowalewska B, Cybulski M, Jankowiak B, KrajewskaKułak E. Acceptance of illness, satisfaction with life, sense of stigmatization, and quality of life among people with psoriasis: a cross-sectional study. Dermatol Ther. 2020;10(3):413-30.

5. Cather JC, Young M, Bergman MJ. Psoriasis and psoriatic arthritis. J Clin Aesthet Dermatol. 2017;10(3):S16-s25.

6. Menter A, Strober BE, Kaplan DH, et al. Joint AADNPF guidelines of care for the management and 
treatment of psoriasis with biologics. J Am Acad Dermatol. 2019;80(4):1029-72.

7. Kemény L, Berggren L, Dossenbach M, Dutronc Y, Paul C. Efficacy and safety of ixekizumab in patients with plaque psoriasis across different degrees of disease severity: results from UNCOVER-2 and UNCOVER-3. J Dermatol Treat. 2019;30(1):19-26.

8. Warren RB, See K, Burge R, et al. Rapid response of biologic treatments of moderate-to-severe plaque psoriasis: a comprehensive investigation using bayesian and frequentist network meta-analyses. Dermatol Ther. 2020;10(1):73-86.

9. Paul C, Griffiths CEM, van de Kerkhof PCM, et al. Ixekizumab provides superior efficacy compared with ustekinumab over 52 weeks of treatment: results from IXORA-S, a phase 3 study. J Am Acad Dermatol. 2019;80(1):70-9.e3.

10. Blauvelt A, Papp K, Gottlieb A, et al. A head-to-head comparison of ixekizumab vs. guselkumab in patients with moderate-to-severe plaque psoriasis: 12-week efficacy, safety and speed of response from a randomized, double-blinded trial. Br J Dermatol. 2020;182(6):1348-58.

11. van der Heijde D, Gladman DD, Kishimoto M, et al. Efficacy and safety of ixekizumab in patients with active psoriatic arthritis: 52-week results from a phase III study (SPIRIT-P1). J Rheumatol. 2018;45(3):367-77.

12. Gordon KB, Blauvelt A, Papp KA, et al. Phase 3 trials of ixekizumab in moderate-to-severe plaque psoriasis. N Engl J Med. 2016;375(4):345-56.

13. Reich K, Pinter A, Lacour JP, et al. Comparison of ixekizumab with ustekinumab in moderate-to- severe psoriasis: 24-week results from IXORA-S, a phase III study. $\mathrm{Br} J$ Dermatol. 2017;177(4): 1014-23.

14. Mease PJ, Smolen JS, Behrens F, et al. A head-tohead comparison of the efficacy and safety of ixekizumab and adalimumab in biological-naïve patients with active psoriatic arthritis: 24-week results of a randomised, open-label, blinded-assessor trial. Ann Rheum Dis. 2020;79(1):123-31.

15. Auguie B. Package 'gridExtra.' https://cran.r-project. org/web/packages/gridExtra/gridExtra.pdf. Accessed 10 May 2021.

16. Wickham H. The Split-Apply-Combine Strategy for Data Analysis. 2011. https://www.jstatsoft.org/ article/view/v040i01. Accessed 10 May 2021.

17. Nakakoji K TA, Yamamoto Y. Cognitive effects of animated visualization in exploratory visual data analysis. In: 5th International Conference on Information Visualisation, 25-26 July 2001; London. https://doi.org/10.1109/IV.2001.942042

18. Crowley JJ. Challenges in psoriasis treatment: nail, scalp, and palmoplantar involvement. In: Weinberg JM, Lebwohl M, editors. Advances in psoriasis: a multisystemic guide. Cham: Springer International Publishing; 2021. p. 343-62.

19. Egeberg A, See K, Garrelts A, Burge R. Epidemiology of psoriasis in hard-to-treat body locations: data from the Danish skin cohort. BMC Dermatol. 2020;20(1):3.

20. Pohl M, Endl H, Fels U. Animated scatterplotanalysis of time-oriented data of diabetes patients. Stud Health Technol Inform. 2016;223:191-8. 